'Oficina de Educación Médica, Facultad de Medicina Universidad Diego Portales. Santiago, Chile. ¿Escuela de Medicina, Facultad de Medicina Universidad Diego Portales. Santiago, Chile. ${ }^{3}$ Oficina de Educación Médica, Facultad de Medicina Universidad Católica del Norte. Coquimbo, Chile.

${ }^{a}$ Químico Farmacéutico. ${ }^{\text {b}}$ Doctor en Ciencias Farmacéuticas.

'Magister en Educación Médica para las Ciencias de la Salud. dEstudiante de Medicina.

Fuente de financiamiento: ninguna.

Los autores declaran no tener conflictos de interés.

Recibido el 19 de diciembre de 2017, aceptado el 25 de julio de 2018.

Correspondencia a: Camilo Torres.

Oficina de Educación Médica, Facultad de Medicina Universidad Diego Portales.

Dirección: Ejército Libertador 141. Santiago, Chile. camilo.torres@udp.cl

\section{Entrustable Professional Activities: Una propuesta innovadora para la evaluación de competencias médicas}

\author{
CAMILO TORRES ${ }^{1}$, LEÓN GOÏTY ${ }^{2, a, b}$, NADIA MUÑOZ ${ }^{3, c}$, PAZ DRAGO ${ }^{2, d}$ \\ Entrustable professional activities: \\ a new proposal for the evaluation \\ of the medical competencies
}

Medical education migrated from a practice-based to a knowledge-based discipline after the publication of the Flexner Report. The emergence of competence-based medical education led to a greater standardization of teaching, allowing students to integrate knowledge, skills and attitudes for the execution of a given task. A challenge is the evaluation of learning. Complex evaluation systems and a consequent atomization that independently assesses different competence components. However, the evaluation carried out at the clinical practice sites allows assessing the overall level of learning. Supervisors observe students' performance and decide if the apprentice can execute a specific task independently. This decision is based upon the trust that the tutor places on the student. Consequently, Ten Cate (2005) proposed the term Entrustable Professional Activities (EPAs), as a framework for professional practice tasks or responsibilities that can be fully entrusted to students, when they demonstrate the competences that are necessary to execute such activity with an increasing level of autonomy.

(Rev Med Chile 2018; 146: 1064-1069)

Key words: Clinical Competence; Education, Medical; Educational Measurement; Professional Competence.
E: n el siglo XX, la publicación del informe Flexner junto con los avances en diagnóstico y tratamiento hicieron que la enseñanza de la medicina pasara de ser una disciplina basada en la práctica, a una disciplina basada en el conocimiento. Hacia finales del siglo XX la gran cantidad de conocimiento de ciencias biomédicas produce un distanciamiento entre el componente teórico y el práctico, lo que se ve reflejado en un aumento de la duración de la carrera y una mayor complejidad de ésta ${ }^{1}$.

Las preocupaciones sobre seguridad del paciente y la responsabilidad que las instituciones educativas tienen en este tema provocaron que las organizaciones profesionales, incluidas las universidades, se dieran cuenta de que la declaración de los resultados de la formación profesional y la evaluación del aprendizaje debían ser más explícitos y estructurados². Por lo tanto, para asegurar una calidad uniforme en los resultados de la formación profesional, los objetivos de aprendizaje no sólo debían incluir conocimientos técnicos, sino que además habilidades profesionales intrínsecas necesarias para trabajar eficazmente en equipos y en el sistema de salud. Para dar respuesta a esta necesidad, a partir del año 1996, distintas organizaciones nacionales (CanMeds, ACGME), plantean perfiles de competencias que los egresados de las especialidades médicas debían tener. Esto generó un 
auge sostenido de la Educación Médica Basada en Competencia (EMBC) ${ }^{3}$.

\section{Educación médica basada en competencia}

La EMBC se define como un paradigma educacional que tiene por propósito generar en los estudiantes las capacidades necesarias para integrar conocimientos, habilidades, destrezas y actitudes, para efectuar una labor determinada $y$ contextualizada ${ }^{4}$.

El desarrollo de la EMBC ha tenido su principal auge a nivel de la educación de postgrado. Sus inicios se remontan a la década de 1960, sin embargo su mayor desarrollo lo ha alcanzado a partir de los años 2000. Durante este período se genera una importante senda en la cual se han podido definir con claridad aspectos relacionados con desarrollo curricular, estrategias de enseñanza y planes de evaluación. Sin embargo, aún existen elementos que requieren una mayor definición, particularmente aquellos relacionados con una estandarización de los conceptos relacionados con competencia y la valoración del aprendizaje, siendo este punto el que se presenta como una de las principales debilidades de la $\mathrm{EMBC}^{3}$.

Existe una importante heterogeneidad ante la definición de competencia, en parte secundario a la multiplicidad de enfoques, perfiles y marco de competencia existentes ${ }^{4}$. Se agrega que la traducción al español de los distintos términos planteados en inglés no es siempre literaria, lo que lleva a una complicación aún mayor.

Englander y cols. ${ }^{5}$, revisaron las definiciones de términos relacionados con competencia, pudiendo estandarizar y precisar los siguientes conceptos:

- Capacidad (competency): Una habilidad observable de un profesional de salud que integra múltiples componentes tales como conocimiento, destrezas, valores, y actitudes. Las capacidades son observables y pueden ser medidas y evaluadas para asegurar su desarrollo.

- Competencia (competence): Es una variedad de habilidades (conocimiento, destrezas y actitudes) a través de múltiples dominios o aspectos del desempeño profesional. Las declaraciones de competencias requieren descriptores que definan las habilidades relevantes, el contexto, y la etapa del entrenamiento. Las competencias son multidimensionales y dinámicas, cambian con el tiempo, la experiencia y el contexto.

- Competente (competent): es la persona que posee las habilidades requeridas en todos los dominios, en un contexto y en una etapa definida de la educación o de la práctica médica.

Dhaliwal y cols. plantean que estos términos están dentro de un continuo; definen capacidad como la habilidad de ejecutar una tarea bien; competente, como aquella persona que puede ejecutar la tarea y competencia como la aptitud de hacer la tarea ${ }^{6}$.

Una de las preocupaciones planteadas por los educadores es lo relacionado a la forma en como se evalúan las competencias. Para responder a esta problemática se han construido sistemas complejos de evaluación del aprendizaje, llevando a la utilización un número importante de instrumentos, y una consiguiente atomización de la evaluación a través de la valoración independiente de los distintos componentes de la competencia. Esta descontextualización de los componentes de la competencia y su baja correlación con las actividades clínicas se presentan como una amenaza para la validez. Con el fin de evitarlo, se prefieren actividades de evaluación que permitan valorar el nivel global de aprendizaje, idealmente efectuadas en el nivel 4 de la Pirámide de Miller ("Hace") Es en este nivel donde la evaluación de las capacidades y competencias se conectan con el ejercicio profesional.

En este contexto, los supervisores determinan si un aprendiz puede ejecutar de forma autónoma o con un grado decreciente de supervisión alguna actividad profesional, dependiendo del nivel de competencia que han alcanzado. Esta decisión que toma el supervisor usualmente se fundamenta en la confianza que deposita en el aprendiz para que este ejecute una tarea ${ }^{8}$.

Esta confianza se debe basar siempre en la observación directa del desempeño del estudiante. La confianza basada en el primer encuentro o la basada en los antecedentes del estudiante (rendimiento académico) no deben ser utilizadas para tomar este tipo de decisiones. Por lo tanto, es la evaluación bien ejecutada por el supervisor de actividades clínicas, la que permitirá determinar si se confía o no en un estudiante para ejecutar una actividad profesional con niveles crecientes de autonomía ${ }^{6}$. 


\section{Actividades profesionales confiables}

Ten Cate plantea que estás actividades profesionales que se confían a los estudiantes para su ejecución con autonomía creciente debían ser conceptualizadas como unidades, proponiendo el término de Entrustable professional activities (EPAs), lo cual en español se traduce como Actividades Profesionales Confiables (APROCs) ${ }^{8}$.

Las EPAs se definen como una unidad de práctica profesional que se puede confiar plenamente a un aprendiz, tan pronto como él o ella haya demostrado la competencia necesaria para ejecutar esta actividad con niveles crecientes de autonomía?.

Definidas originalmente para educación de posgrado (especialidades médicas) las EPAs han tenido una rápida difusión sobre todo en países donde existen marcos nacionales de competencias para educación de post-grado ${ }^{6}$. Como se observa en la Tabla 1, para que una actividad profesional se considere como EPA, debe cumplir características particulares?.

Es importante recalcar que las EPAs se diferencian de las competencias, en que estas ${ }^{10}$ :

- No son una alternativa de las competencias, sino una forma de traducirlas en actividades profesionales.

- Las competencias son descriptores de habilidades complejas de los profesionales, las EPAs son descriptores de actividades profesionales.

- Las EPAs requieren de la integración y ejecución de varias competencias de forma simultánea.

La ventaja de las EPAs es que operacionalizan las competencias a través de la vinculación con las actividades profesionales. Esto permite a los supervisores y estudiantes focalizarse en la evaluación de actividades clínicas mediantes la utilización de instrumentos ad-hoc, por ejemplo el minicex. Al realizar la evaluación de las EPAs los supervisores valoran el desempeño de los estudiantes en la realización de actividades para lo cual requerirán simultáneamente la utilización de varias competencias 9 .

Con el fin de certificar que, en base a la confianza otorgada por el tutor, un estudiante ha pasado el umbral que le permite la obtención de autonomía en una actividad, se plantea que este acto sea reconocido mediante una declara- ción de responsabilidad otorgada (statement of awarded responsability; STAR). En la medida que el estudiante avance en su formación y vaya consiguiendo un número mayor de declaraciones de responsabilidades otorgadas para distintas EPAs, mayor irá siendo su nivel de competencia y de autonomía $^{10}$.

Como se describe en la Tabla 2, a medida que el aprendiz tenga más declaraciones de responsabilidad otorgada, irá teniendo distintos niveles de autonomía ${ }^{11}$.

Para la construcción de las EPAs no basta solamente con realizar un listado de las actividades que debe ejecutar un profesional en el día a día, sino que además debe contener elementos que permitan su utilización para guiar la evaluación ${ }^{12}$, estos son:

\section{Tabla 1. Características para que una actividad profesional sea considerada EPA (Ten Cate \& Scheele, 2007)}

1. Son parte del trabajo profesional esencial ejecutado en un contexto determinado

2. Requieren conocimientos, destrezas y actitudes, adquiridos durante la formación

3. Deben conducir a la formación necesaria para la ejecución de las labores profesionales

4. Son confinadas a personal cualificado

5. Se deben ejecutar de forma independiente y dentro de un marco de tiempo

6. Deben ser observables y medibles en su proceso y en su resultado, conduciendo a una conclusión (logrado o no logrado)

7. Son el reflejo de una o más de las capacidades que debe desarrollar un estudiante

Tabla 2. Niveles de autonomía alcanzables por el aprendiz (Ten Cate, 2010)

1. Está presente y observa

2. Actúa con supervisión directa y proactiva. i.e con un tutor presente de forma física en la sala

3. Actúa con supervisión indirecta, reactiva. i.e tutor disponible ante consulta

4. Actúa sin supervisión

5. Supervisa a estudiantes más novatos 
1. Título: Debe ser conciso e informativo. Como refleja una actividad de trabajo, no debe ser planteado como resultado/objetivo. Idealmente debe tener máximo 10 palabras.

2. Especificaciones y limitaciones: Las especificaciones deben señalar de forma precisa qué se incluye y excluye en la actividad. Además debe incluir el contexto y el nivel de desarrollo profesional (pregrado, postgrado, educación continua).

3. Dominios de competencia más relevantes: Esta sección relaciona la EPA con el marco de competencia utilizado. Se deben mencionar los dominios de competencia o capacidades que se necesitarán para realizar la EPA, para esto es necesario construir una matriz de EPAs-competencias.

4. Conocimientos, destrezas y actitudes necesarios para ejecutarla: Los estudiantes deben saber cuáles son los conocimientos, destrezas $\mathrm{y}$ actitudes que se esperan, previo a que se les de autonomía. También es importante que conozcan la experiencia (tipo de rotación, tipo de paciente, números de procedimientos) previa que deben haber tenido para obtener la autonomía.

5. Fuentes de información para evaluar progreso: Describir que instrumentos se usarán para obtener la información que permita determinar el progreso del estudiante.

6. Indicar de acuerdo al nivel de avance curricular del estudiante, qué nivel de autonomía tendrá para ejecutar la actividad.

7. Fecha de expiración: Es opcional, se usa para establecer un período de revalidación. Las EPAs se mantienen vigentes solo si se mantienen las competencias necesarias para ejecutarlas.

La Tabla 4 muestra un ejemplo de EPA con cada uno de sus elementos.

\section{EPAs en el pregrado}

Las EPAs fueron planteadas inicialmente para el uso a nivel de postgrado. Sin embargo, la utilización de éstas también es factible de realizar a nivel de pregrado. La educación médica de pregrado se caracteriza por ser una etapa dentro del continuo formativo (pregrado, especialidad, educación continua), en la que un individuo debe desarrollar capacidades y adquirir competencias específicas para ejecutar tareas dentro de su ámbito de acción ${ }^{13}$.

Además, el declarar EPAs en la formación de pregrado permite que éstas se vinculen con las EPAs que los estudiantes deben realizar al finalizar el nivel de postgrado. Los niveles de autonomía crecientes declarados en las EPAs se alinean con el modelo de Dreyfus \& Dreyfus y bajo esta visión, la adquisición de las capacidades y el desarrollo de competencias es un proceso continuo que trasciende a las distintas etapas formativas ${ }^{11}$.

Por lo tanto, a nivel de pregrado se espera que para ciertas EPAs el estudiante llegue hasta un nivel de autonomía determinado, mientras que para otras debe ejecutarlas completamente de forma autónoma. Las variables en las que el supervisor se sustenta para tomar la decisión de una declaración de responsabilidad otorgada son las mismas que a nivel de postgrado ${ }^{13}$.

El contar con EPAs en pregrado permitiría delimitar de manera más precisa cuál es el nivel de responsabilidad que el estudiante va adquiriendo a medida que avanza en la formación profesional. Además pueden articular la actividad de aprendizaje en el lugar de práctica clínica con la contribución que el estudiante realiza a la atención del paciente, permitiendo de esa forma otorgar un rol al alumno, explicitar la contribución a la atención clínica y promover una mayor seguridad del paciente ${ }^{13}$.

Uno de los puntos críticos relacionados con la utilización de las EPAs hace relación con la cantidad y el nivel de especificidad que deberían poseer. A nivel de postgrado se plantea que un número apropiado para una especialidad debería de estar en el orden de 20 a 30, a este nivel las EPAs son actividades complejas, para lo que se requiere posesión de capacidades y competencias de nivel alto ${ }^{14}$.

Tabla 3. Componentes de EPAs adaptados a la realidad canadiense (AFMC, 2016)

1. Título

2. Descripción

3. Dominios de competencia

4. Comportamientos pre y post entrega de autonomía en base a la confianza

5. Fuentes de información para evaluar progreso 
Tabla 4. Ejemplo de EPA según la AFMC (AFMC, 2016)

\begin{tabular}{|c|c|c|}
\hline Título & \multicolumn{2}{|c|}{ Presentación de informes orales y escritos que documentan un encuentro clínico } \\
\hline Descripción & \multicolumn{2}{|c|}{$\begin{array}{l}\text { El graduado presenta un resumen conciso y relevante, incluyendo los aspectos positivos y negativos } \\
\text { de un encuentro clínico, a los miembros del equipo (incluidos los pacientes, y cuándo es legalmente } \\
\text { relevante, miembros de la familia) facilitando la atención continua. Él/ella sigue la legislación vigente } \\
\text { y toma en consideración aspectos éticos del quehacer profesional }\end{array}$} \\
\hline $\begin{array}{l}\text { Dominios de } \\
\text { competencia }\end{array}$ & \multicolumn{2}{|l|}{$\begin{array}{l}\text { Experto médico } \\
\text { Comunicación } \\
\text { Colaborador } \\
\text { Profesionalismo }\end{array}$} \\
\hline \multirow{2}{*}{$\begin{array}{l}\text { Comportamientos } \\
\text { pre y post entrega } \\
\text { de autonomía en } \\
\text { base a la confianza }\end{array}$} & Pre & Post \\
\hline & $\begin{array}{l}\text { El estudiante: } \\
\text { - Presenta un resumen desenfocado, } \\
\text { inexacto, desorganizado y sin informa- } \\
\text { ción importante } \\
\text { - No demuestra una reflexión comparti- } \\
\text { da entre el paciente, los miembros del } \\
\text { equipo de atención médica y consulto- } \\
\text { res } \\
\text { - Documenta los hallazgos de manera } \\
\text { poco clara }\end{array}$ & $\begin{array}{l}\text { El estudiante: } \\
\text { - Presenta un reporte conciso, resumido y considerando } \\
\text { los elementos relevantes del encuentro con el paciente } \\
\text { - Toma consideración en su presentación de los aspec- } \\
\text { tos relacionados con la visión del paciente y fu familia } \\
\text { - Especifica en el informe el contexto del paciente } \\
\text { - Demuestra una reflexión compartida entre el paciente, } \\
\text { miembros del equipo respecto al cuidado de salud del } \\
\text { paciente } \\
\text { - Documenta los hallazgos de una manera clara, enfo- } \\
\text { cada y precisa }\end{array}$ \\
\hline $\begin{array}{l}\text { Fuentes de } \\
\text { información para } \\
\text { evaluación }\end{array}$ & \multicolumn{2}{|c|}{$\begin{array}{l}\text { Esta EPA debe evaluarse mediante observación directa en diversos contextos clínicos (incluidos enfer- } \\
\text { medades médicas agudas y crónicas), en pacientes de varios grupos de edad incluidos los niños y sus } \\
\text { padres, adultos y ancianos. Además, se puede evaluar utilizando pacientes simulados y / o exámenes } \\
\text { clínicos objetivos estructurados También se puede evaluar mediante la revisión de historias clínicas }\end{array}$} \\
\hline
\end{tabular}

A nivel de pregrado se plantea la alternativa de utilizar EPAs similares a las de postgrado, pero limitando el nivel de autonomía del estudiante. Para eso es necesario que el nivel de detalle de los niveles de autonomía sea mayor. Otra alternativa hace relación con la consideración de hitos en el desarrollo de competencias, y que estos se vinculen con los niveles de autonomía ${ }^{13}$.

En el año 2009 el Accreditation Council for Graduate Medical Education (ACGME) planteó la creación de hitos (milestones), los cuales son descriptores de un desempeño determinado y específico de acuerdo al nivel de desarrollo de competencias del estudiante en las especialidades médicas. Estos fueron desarrollados con el propósito de lograr un mejor nivel de la evaluación del desempeño. La creación de los hitos ha permitido contar con una descripción de las capacidades en términos de comportamientos observables que tengan sentido para los profesores y los estudiantes, el actuar como una hoja de ruta de aprendizaje para los estudiantes, y el servir como una fuente para retroalimentación y evaluación ${ }^{3}$.

A pesar de las ventajas de los hitos para la eva- luación del aprendizaje en EMBC, éstos no logran evaluar la integración de capacidades a través de dominios de competencia, aspecto fundamental para certificar la independencia en la actividad profesional. Una de las formas que permiten superar esta situación es a través de la utilización las actividades profesionales delegables, como elementos integradores para permitir evaluación integral de las competencias ${ }^{15}$.

La American Associaton of Medical College (AAMC) en el año 2013, llevó adelante un proyecto para determinar las EPAs esenciales que el médico recién egresado debía realizar al momento de ingresar a la especialidad. Para llevar a cabo este proceso, combinaron el modelo de las EPAs, capacidades (competencies), e hitos (milestone). El resultado de esto fue la determinación de 13 EPAs con sus respectivos dominios de competencias, capacidades e hitos. Este documento fue creado con el propósito de estandarizar a nivel nacional las actividades de formación profesional que las Escuelas de Medicina en Estados Unidos realizan ${ }^{16}$.

La propuesta planteada por esta asociación puede ser considerada como una forma de abordar 
las EPAs a nivel de pregrado. Otra alternativa es la planteada por Chen y cols. ${ }^{13,17}$, la cual hace relación con considerar una escala distinta de niveles de autonomía para los estudiantes de pregrado, así como también un mayor detalle de las diferentes competencias necesarias para la ejecución de la EPAs.

En el año 2016 la Association of Faculties of Medicine of Canada (AFMC) presentó las EPAs de transición entre la Escuela de Medicina y la Residencia. En este proyecto se revisaron y adaptaron EPAs planteadas por distintas instituciones (AAMC, Universidad de Ultrech) a la realidad canadiense, haciendo el mapeo con el marco de competencia CanMeds y estableciendo los componentes de estas (Tabla 3$)^{18}$.

Tanto las alternativas de EPAs para el pregrado propuestas por la AAMC, AFMC y por Chen y colaboradores, se presentan como posibles para desarrollar y un mayor nivel de estudios será necesario para demostrar cual es mejor.

La EMBC ha alcanzado un nivel de desarrollo que permite dar respuesta al fin último de la educación médica, que es el formar un médico que provea de servicios de cuidados al paciente con un alto nivel de calidad. La definición de las EPAs permite que la certificación de las competencias del médico, se efectúe de forma más fidedigna, y de esta forma colabora a la formación profesional ${ }^{15}$. Actualmente constituye una propuesta educativa clara, innovadora y útil, que debiera considerarse tanto a nivel macro como microcurricular, para aunar criterios en la formación médica y asegurar la calidad profesional.

\section{Referencias}

1. Ten Cate O, Tobin S, Stokes M. Bringing competencies closer to day-to-day clinical work through entrustable professional activities. Med J Aust 2017; 206 (1): 14-6.

2. Kohn LT, Corrigan JM, Donaldson MS. To err is human. Building a safer health system. 2000. Washington, DC: National Academy Press.

3. Carraccio C, Englander R. From Flexner to competencies: reflections on a decade and the journey ahead. Acad Med 2013; 88 (8): 1067-73.

4. Frank JR, Snell LS, Cate OT, Holmboe ES, Carraccio C, Swing S, et al. Competency-based medical education: Theory to practice. Med Teach 2010; 32 (8): 638-45.

5. Englander R, Cameron T, Ballard AJ, Dodge J, Bull J,
Aschenbrener CA. Toward a common taxonomy of competency domains for the health professions and competencies for physicians. Acad Med 2013; 88 (8): 1088-94.

6. Dhaliwal U, Gupta P, Singh T. Entrustable Professional Activities: Teaching and Assessing Clinical Competence. Indian Pediatrics 2015; 52: 591-7.

7. Van der Vleuten CPM, Schuwirth LWT. Assessing professional competence: from methods to programmes. Med Educ 2005; 39 (3): 309-17.

8. Ten Cate O. Entrustability of professional activities and competency based training. Med Educ 2005; 39 (12): 1176-7.

9. Ten Cate O, Scheele F. Competency-based postgraduate training: Can we bridge the gap between theory and clinical practice? Acad Med 2007; 82 (6): 542-7.

10. Ten Cate O. Nuts and bolts of entrustable professional activities. J Grad Med Educ 2013; 5 (1): 157-8.

11. Ten Cate O, Snell L, Carraccio C. Medical competence: the interplay between individual ability and the health care environment. Med Teach 2010; 32 (8): 669-75.

12. Mulder H, Ten Cate O, Daalder R, Berkvens J. Building a competency-based workplace curriculum around entrustable professional activities: The case of physician assistant training. Med Teach 2010; 32 (10): e453-9.

13. Chen HC, van den Broek WES, Ten Cate O. The case for use of entrustable professional activities in undergraduate medical education. Acad Med 2015; 90:431-6.

14. Ten Cate O, Chen HC, Hoff RG, Peters H, Bok H, van der Schaaf M. Curriculum development for the workplace using Entrustable Professional Activities (EPAs): AMEE Guide No. 99. Med Teach 2015; 37(11), 9831002.

15. Carraccio C, Englander R, Gilhooly J, Mink R, Hofkosh D, Barone MA, et al. Building a Framework of Entrustable Professional Activities, Supported by Competencies and Milestones, to Bridge the Educational Continuum. Acad Med 2017; 92 (3): 324-30.

16. Association of American Medical Colleges (AAMC). Core entrustable professional activities for entering residency. 2013. https://www.mededportal.org/icollaborative/resource/887.

17. Chen HC, McNamara M, Teherani A, Ten Cate O, O’Sullivan P. Developing Entrustable Professional Activities for Entry Into Clerkship. Acad Med 2016; 91 : 247-55.

18. Association of Faculties of Medicine of Canada (AFMC). Entrustable Professional activities for the transition from medical school to residency. 2016.https://afmc.ca/ sites/default/files/documents/AFMC_Entrustable_Professional_Activities_EN_0.pdf. 